\title{
The growth of $B$ cell receptor microcluster is a universal response of $B$ cells encountering antigens with different motion features
}

\author{
Zhengpeng Wan, Wanli Liu \\ School of Life Sciences, Tsinghua University, Beijing 100084, China \\ $\triangle$ Correspondence: liuwanli@biomed.tsinghua.edu.cn \\ Received June 3, 2012 Accepted June 15, 2012
}

\begin{abstract}
$B$ lymphocyte cell senses and acquires foreign antigens through clonal distributed $B$ cell receptors (BCRs) expressed on the surface of plasma membrane. The presentation formats of antigens are quite diverse. Based on their Brownian diffusion mobility, there are three forms: free mobile soluble antigens, lateral mobile membrane bound antigens, and fixed immobile antigens. Here, using high resolution high speed live cell imaging approaches, we provide evidence that BCR microclusters are formed on the surface of $B$ cells shortly after B cell's encountering of antigens with each format of motion features. Through high speed live cell imaging, we determine that these BCR microclusters show dynamic growth feature and by doing so function as the basic platforms for $B$ cells to acquire the antigens. We propose that the formation and dynamic growth of BCR microcluster is a universal mechanism for $B$ cell to response to antigens with diverse motion features.
\end{abstract}

KEYWORDS BCR microcluster, TIRFM, live cell imaging, fixed immobile antigen, lateral mobile antigen, free mobile antigen

\section{INTRODUCTION}

B cells constitute one of the two arms in adaptive immune system, being responsible for the antibody response. On sensing cognate antigens, B cells get activated, proliferate and differentiate to plasma cells and memory $B$ cells (Pierce and Liu, 2010). All these complicated and ordered B cell re- sponses start with the B cell's sense and subsequent acquisition of foreign antigens. Thus it is very important to investigate the molecular mechanism of how $B$ cells acquire antigens. It is well established that as an initial step $B$ cells sense the antigens through the surface expressed BCRs (or, antigen receptors). The $\mathrm{B}$ cell receptor (BCR) is composed of a membrane form of the $\mathrm{lg}(\mathrm{mlg})$ and noncovalently associated heterodimer of Ig $\alpha$ and $\lg \beta$ in a $1 \mathrm{mlg}: 1 \lg \alpha-\lg \beta$ heterodimer stoichiometry (Schamel and Reth, 2000; Tolar et al., 2005). In contrast, the counterparts of BCRs, the antigens, can show great diversity based on their presentation formats. Many early studies demonstrated that the presentation format of antigens can dramatically influence the outcome of antibody responses (Bachmann et al., 1993). The presentation format of antigens can be divided into different categories depending on the criteria. In this study, we focus on the state of the Brownian diffusion mobility of antigens (Kusumi et al., 2005, 2010). Under these criteria, there are at least three presentation formats of antigens: free mobile soluble antigens, lateral mobile membrane bound antigens, and fixed immobile antigens. Free soluble antigens can do three dimensional $(X, Y$ and $Z$ ) free Brownian motion; lateral mobile membrane bound antigens can do two dimensional $(X$ and $Y$, but not $Z$ ) free Brownian motion; while fixed immobile antigens cannot do Brownian motion in each dimension (Kusumi et al., 2005, 2010). All three types of antigens formats have been used in $B$ cell biology studies. For example, the well-established BCR signaling cascades were actually demonstrated by stimulating the B cells with multivalent soluble antigens in solution, which are typical free mobile soluble antigens (Kurosaki et al., 2010; Reth and Wienands, 1997).

The lateral mobile membrane bound antigens are currently believed to be the major formats of the antigens that $B$ cells will encounter in vivo (Batista and Harwood, 2009). Batista 
and colleagues first showed by confocal imaging that B cells could be more efficiently activated by antigens tethered on the surface of antigen presenting cells (APCs) compared to soluble antigens (Batista et al., 2001). Subsequently, several intravital imaging studies provided evidence that most antigens including viruses and immune complexes are mainly captured by macrophages or dentritic cells lining the subcapsular sinuses and are transported into the cortex of the lymph node when they were presented to $B$ cells (Carrasco and Batista, 2007; Junt et al., 2007; Phan et al., 2007; Qi et al., 2006). When antigens are captured and tethered on the surface of the plasma membrane, by nature, these antigens generally lose their ability to do free Brownian motion at the $Z$ dimensional, and are thus termed as lateral mobile membrane bound antigens.

The lateral mobile membrane bound antigens are the format of antigens drawing most of the attention of $B$ cell immunologists in the past years. Using high resolution live cell imaging techniques, Fleire et al. (2006) provided the first detailed description of the molecular events that follow the $\mathrm{B}$ cell's encounter with antigen containing fluid planar lipid bilayers in vitro as a surrogate for APCs. They observed that B cells dramatically spread over the lipid bilayer to search for the cognate antigens. As the B cells spread, BCR microclusters formed in the peripheral lamellopodia of the cell. After maximal spreading, the B cells contracted to form an ordered immune synapse. This remarkably dynamic two phase spreading and contraction response of the B cells was the first ever captured events happened on the plasma membrane to visualize how $B$ cells acquire membrane bound antigens (Fleire et al., 2006). The observation that BCRs firstly formed microclusters steps before the formation of immunological synapse (IS) suggests that the BCR microclusters are the most elementary platform for the B cells to sense and acquire antigens. Indeed, in our recent studies, using a variety of cutting-edge high resolution high speed live cell and single molecule imaging techniques supported by Total Internal Reflection Fluorescence Microscopy (TIRFM), we have shown that upon $B$ cell's recognition of lateral mobile membrane bound antigens tethered on the planar lipid bilayers, the BCR microcluster can become bigger and brighter over time, a process we termed as BCR microcluster growth (Liu et al., 2010a). We determined that the growth of BCR microclusters is a fundamental step for $B$ cells to acquire antigens. Moreover, it is widely observed that BCR microcluster is a basic platform for $B$ cells to initiate BCR signaling (Batista and Harwood, 2009; Pierce and Liu, 2010; Treanor and Batista, 2010).

The nature of the antigens the $B$ cells encounter is quite diverse. The membrane bound antigens are just one of the three types of antigens under a category defined by the Brownian mobility feature of antigens. It is not clear whether or not the BCR microclusters will form when B cells encounter the other types of antigens? If the answer is yes, it is not clear whether or not the BCR microclusters will show the dynamic growth feature and by doing so make it as the fundamental platform for B cells to acquire antigens. To answer these questions, using high resolution and high speed live cell imaging approaches, we provide evidence here that BCR microclusters are formed on surface of B cells shortly after B cell's encountering of each types of antigens. Through time lapse imaging, we determined that these BCR microclusters show dynamic growth feature. All these molecule events help the $B$ cells acquire antigens. We propose that the formation and dynamic growth of BCR microcluster is a universal mechanism for $B$ cell to response to the antigens with diverse motion features.

\section{RESULTS}

\section{The response of $B$ cells encountering fixed immobile antigens}

We first examined the response of Ramos B cells when encountering antigens with different motion features. Ramos is a human Burkitt's lymphoma B cell line expressing human IgM-BCRs. We used goat anti-human IgM antibodies as surrogate antigens to stimulate Ramos B cells. Anti-human IgM antibodies were coated on the acid cleaned glass cover-slide. The antigen in this format served as the fixed and immobile antigen. We stained the BCRs on Ramos cells with Alexa568 conjugated Fab fragment anti-human IgM constant region specific antibodies (Fab-Alexa568 anti-human IgM), which are able to efficiently label the IgM-BCRs while avoiding the potential of having the $B$ cells activated. We loaded these pre-labeled Ramos B cells on the cover-slides coated with or without antigens. The behavior of the $B$ cells and the dynamics of BCR microclusters were examined every $2 \mathrm{~s}$ beginning with the initial contact of the cells with the slides by collecting Alexa568 TIRFM images (Fig. 1; Supplemental video 1). By doing so, we are able to examine in real time and in live cells the B cell's capability of acquiring fixed immobile antigens through its accumulating the antigen-engaged BCRs at the interface of the B cell's contact with the cover-slides.

When placed on antigen coated cover-slides, the Ramos cells initially touched the antigen-coated slides through several of its membrane protrusions, and BCR microclusters formed shortly after $B$ cell's encountering of these fixed immobile antigens (Fig. 1A; Supplemental video 1). Moreover, the BCR microclusters obviously become bigger and brighter over time, suggesting that more and more BCRs accumulated into these microclusters (Fig. 1A, video 1). We next characterized the growth of individual BCR microclusters by analyzing the time lapse TIRFM images using the 2D Gaussian function fitting as reported (Liu et al., 2010a). The growth of the BCR microclusters was quantified by both size (the diameter of the fluorescence spot in TIRFM images) and fluorescence intensity ( $\mathrm{Fl}$ of the fluorescence spot). To ac- 
A

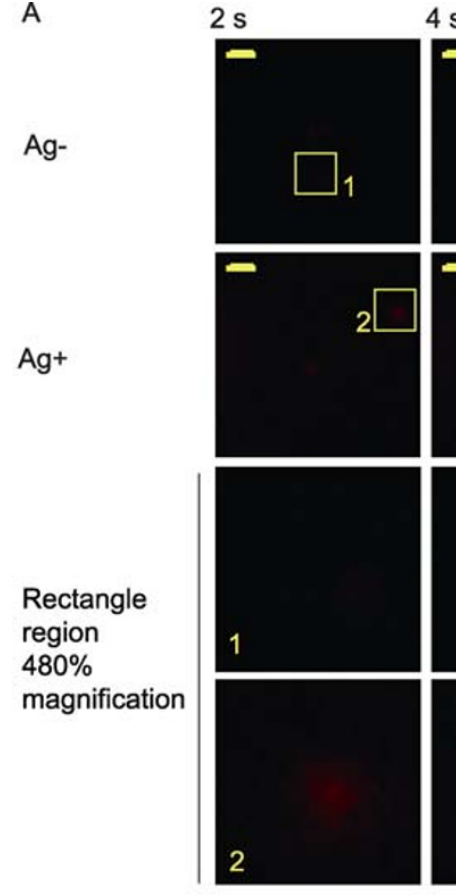

$4 \mathrm{~s}$
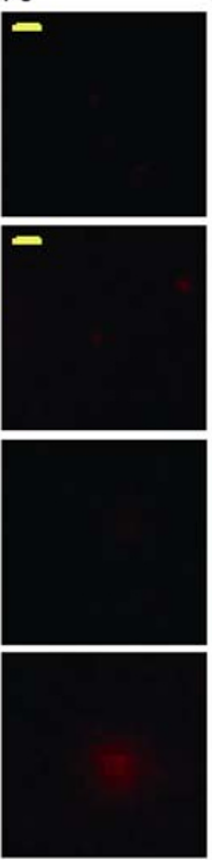

$8 \mathrm{~s}$
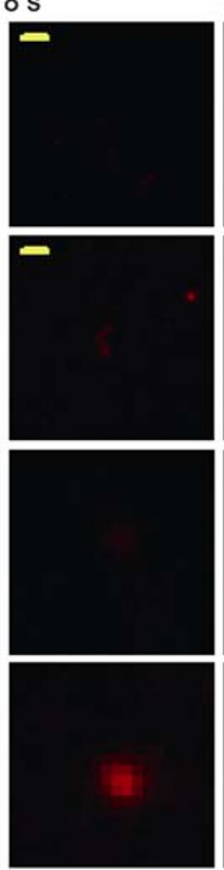

$16 s$
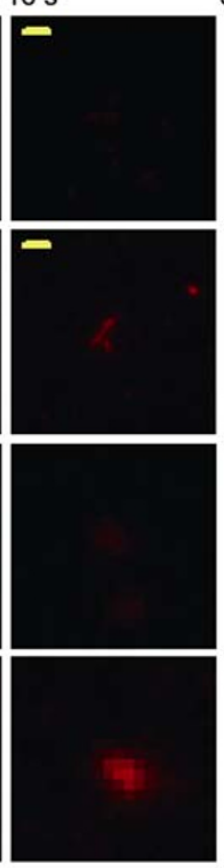

$32 \mathrm{~s}$
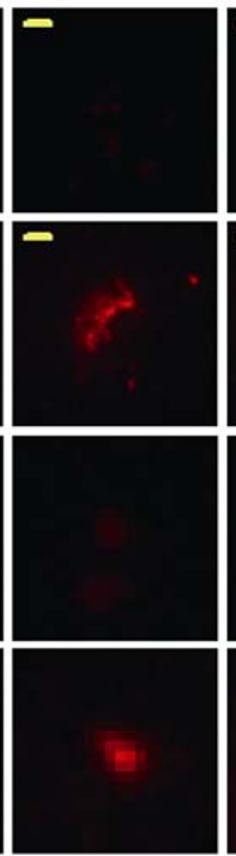

$60 \mathrm{~s}$

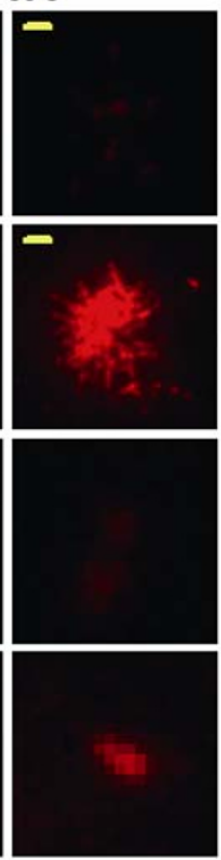

B

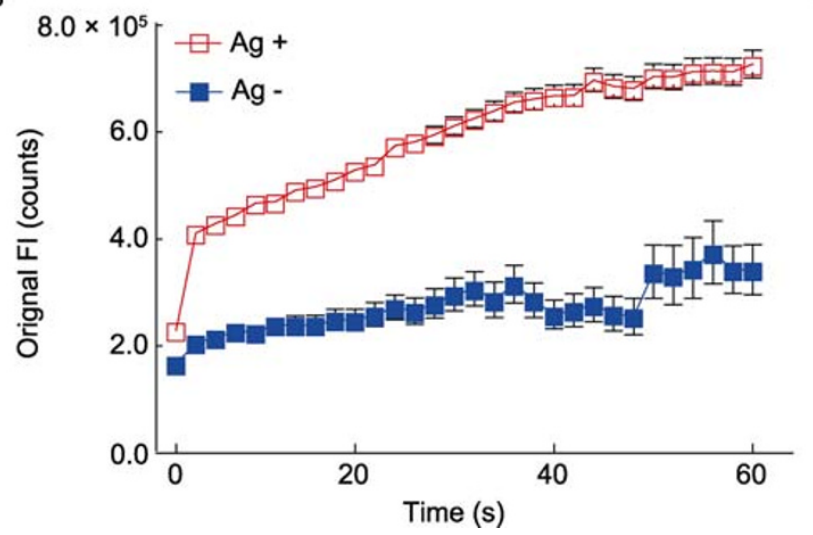

C

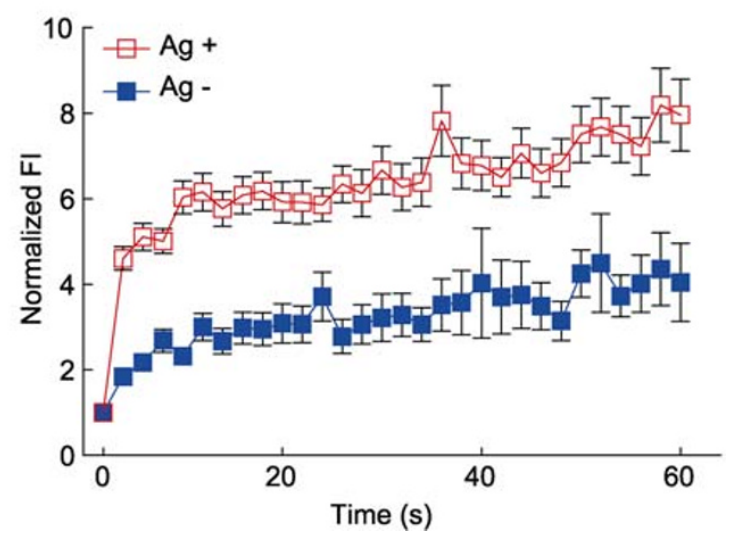

Figure 1. The response of Ramos cells encountering fixed immobile antigens. (A) Given are TIRF images of Ramos cells that were pre-labeled with Alexa568-Fab anti-IgM and were placed on the cover slides coated with or without antigens at the indicated time points. Time lapse movie is given in Supplemental video 1. Scale bar is $1.6 \mu \mathrm{m}$. Typical microclusters in the images indicated by the yellow box 1 and box 2 are shown at $480 \%$ magnification for better resolution. (B and $C$ ) Shown are the original (B) and normalized FI (C) of BCR microclusters examined by Alexa568-Fab anti-IgM TIRFM images. The data represent means \pm SEM of at least 15 thousands BCR microclusters acquired from three independent experiments.

curately quantify these two parameters of all BCR microclusters at each time point, each BCR microclusters was analyzed by fitting them individually to a two-dimensional (2D) Gaussian function (Holtzer et al., 2007). By doing this, we are able to use the parameters yielded from the 2D Gaussian function to mathematically quantify the BCR microclusters. For each BCR microcluster, the $x$ and $y$ coordinates define the position of BCR microclusters, the integrated FI defines the amount of BCRs accumulated into each BCR microcluster, while the full width at half-maximum peak height (FWHM) of the intensity distribution in the $\mathrm{x}$ and $\mathrm{y}$ direction tells the size of BCR microclusters. To minimize tracking and Gaussian fitting errors that will arise from microcluster merging and overlapping at later time points, we only focus on the first $60 \mathrm{~s}$ of each track of the microclusters.

Typical examples of the growth of BCR microclusters from $B$ cells placed on cover-slides coated with or without antigens in the imaging time course are depicted (Fig. 1B). It is quite obvious that the Ramos cells form BCR microclusters at the initial contact points between the cells and the cover-slides. 
These clusters obviously grew in FI and size with time. The growth of the BCR microclusters with time was antigen dependent, as stable clusters did not form or grow on antigen free cover-slides (Fig. 1B). By analyzing the FI of hundreds of BCR microclusters produced on antigen-coated slides, we determined that the increase in $\mathrm{FI}$ of individual clusters to be rapid, with the $\mathrm{Fl}$ increasing 8-fold over $60 \mathrm{~s}$ (Fig. 1B and 1C). However, we did observe a two to three fold increase in $\mathrm{FI}$ from the Ramos B cells placed on cover-slides without antigens. This increase in $\mathrm{FI}$ may simply reflect the increase in $\mathrm{FI}$ with enhanced illumination in the TIRF field as the B cell approaches the cover-slides; or represent an antigen-indepen-

A

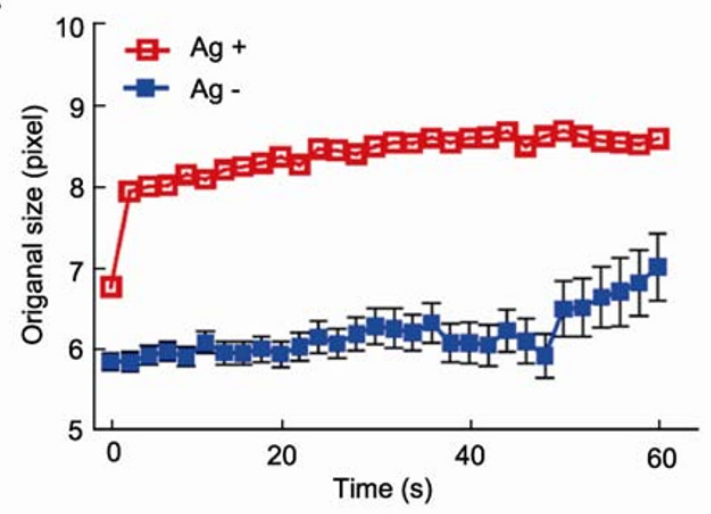

dent accumulation of BCRs in the first contact points between the cells and the cover-slides, or a mixture from both effects.

We are also curious on the change of the size (diameter) of the BCR microclusters produced on B cells encountering the fixed immobile antigens coated on cover-slides. Examples of the increase in microcluster size imaging Fab-Alexa568 anti-human IgM for the Ramos B cells placed on cover-slides coated with or without antigens in the imaging time course are depicted (Fig. 1A). A statistical comparison from hundreds of BCR microclusters indicated that in the $60 \mathrm{~s}$, the microclusters increased 1.4 folds in size in an antigen-dependent manner (Fig. 2A and 2B). We also examined

B

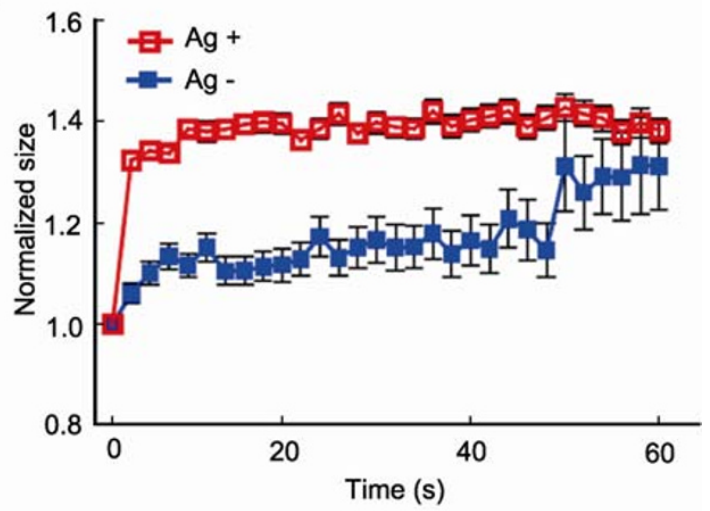

C

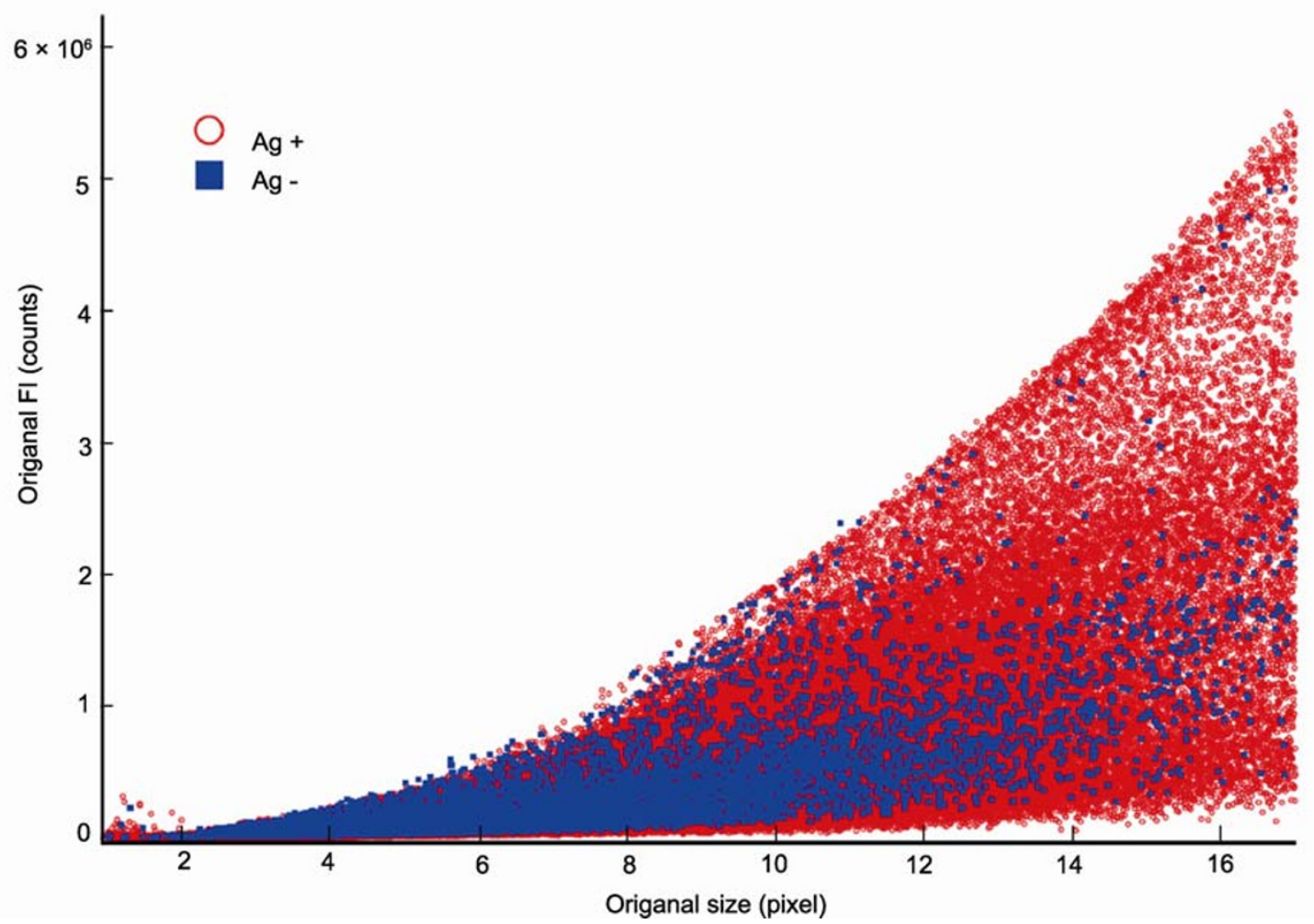

Figure 2. BCR microclusters grow in size with time when encountering fixed immobile antigens. (A and $B$ ) Shown are the changes of the original $(A)$ and normalized $(B)$ size of BCR microclusters of Ramos cells placed on cover slides coated with or without antigens. The data represent means \pm SEM of at least 15 thousands BCR microclusters acquired from three independent experiments. (C) Correlation of $\mathrm{FI}$ and size of all the BCR microclusters. 
the correlation of the $\mathrm{FI}$ and the size of the BCR microclusters. A linear regression of the paired values of $\mathrm{FI}$ and the size of all the BCR microclusters over the entire time course showed a strong positive correlation between the $\mathrm{FI}$ and the size of BCR microclusters (Fig. 2C). This indicates that BCR microclusters grow proportionately in size and in the number of BCRs in the clusters.

These analyses focusing on the very early behaviors (60 s) of BCR microclusters indicate that Ramos B cells can very well distinguish the presence of fixed immobile antigen coated on cover-slides and start to accumulate antigens seconds after the BCR-antigen engagement. We also want to confirm these observations by analyzing response of $B$ cells at some later time points. Here we examined by TIRFM the accumulation of BCRs into the B cell's contact with the cover-slides $10 \mathrm{~min}$ after loading the $\mathrm{B}$ cells on the cover-slides (Fig. 3A). The amount of BCRs accumulated into the $B$ cell's contact with the cover-slides and the size of $B$ cell's contact area were quantified by image pro software. Indeed we are able to determine that Ramos B cells encountering the antigen-coated cover-slides dramatically accumulated much more amount of BCRs into the cells' contact with the slides (Fig. 3B). When quantifying the $B$ cell's spread over on the antigen coated cover-slides, we found that Ramos B cells showed much bigger contact area (Fig. 3C). All these observations were not observed or very weak for the Ramos B cells placed on the cover-slides without antigen (Fig. 3). To exclude the bias of TIRFM images on this type of comparisons, we also examined the distribution of BCRs on resting $B$ cells versus the $B$ cells encountering the immobile antigens fixed on glass cover slides by spinning disk confocal microscope (Fig. 3D; Supplemental video 2). As shown in the reconstructed 3D images and in the video, it is quite clear the BCRs are evenly dispersed on the cell surfaces in resting $B$ cells, while antigen-primed BCRs are obviously accumulated and polarized towards to the cell's contact with the antigen-coated cover slides (Fig. 3D), consistent with the results from TIRFM images. To make sure that these observations are of general meanings, we also used the primary splenic B cells from B1-8 transgenic mice and imaged the behaviors of these primary $B$ cells when placed on cover-slides coated with B1-8-BCR specific antigens NIP25-BSA (Fig. 4). Indeed, when encountering the fixed immobile NIP25-BSA antigens, B1-8 B cells showed much larger contact area and a large degree of accumulating BCRs into the cells' contact interface with the cover-slides compared to the case of antigen-free cover-slides (Fig. 4B and 4C).

Collectively, these results show that the fixed immobile antigens coated on the surface of cover-slides can strongly stimulate the B cells to spread much broader to search for more antigens, and to accumulate BCRs into the cell's contact interface, suggesting the presentation format of fixed immobile antigens can serve as good BCR stimulus.

\section{The response of $B$ cells encountering lateral mobile antigens}

The responses of B cells and BCRs when encountering the lateral mobile antigens have been well studied by others and us recently (Fleire et al., 2006; Depoil et al., 2008; Lin et al., 2008; Weber et al., 2008; Tolar et al., 2009b; Liu et al., 2010a, b, c, d; Pierce and Liu, 2010; Treanor and Batista, 2010; Treanor et al., 2010). Here, we thus just use the imaging and quantification of the $B$ cells response to this presentation format of antigens as a control of our images acquisition condition and analyses. Consistently well with the published studies, we are able to demonstrate that the BCR microclusters on Ramos B cells when placed on lipid bilayers containing antigens grow dramatically in both $\mathrm{FI}$ and size compared to the case of B cells placed on lipid bilayer control (Fig. 5A-5E). Subsequently, at 10 min time point, the Ramos $B$ cells accumulated much more amount of BCRs into the cells' contact interface with the antigen-containing lipid bilayers, and showed much bigger contact area, compared to the $B$ cells placed on antigen free control lipid bilayers as quantified by TIRFM images (Fig. 6A-6C). Similar observations were got when imaging the response of B1-8 primary $B$ cells placed on fluid planar lipid bilayers containing NIP1-His12 antigens compared to the case of B1-8 $\mathrm{B}$ cells placed on antigen free planar lipid bilayers (Fig. 6D-6F).

\section{The response of $B$ cells encountering free mobile soluble antigens}

The free mobile soluble antigen is probably the most commonly used format of antigen in B cell biology studies. This type of antigen shows some very unique features that do not possess by any other formats of antigens (Kusumi et al., $2005,2010)$. Each antigen molecule of free mobile soluble antigen can do 3-dimensional free Brownian motion in solution. Early biochemical studies use this type of antigens well addressed the BCR signaling pathway molecules (Kurosaki et al., 2010; Reth and Wienands, 1997). In these biochemical experiments, the amount of antigens was usually sufficient, thus most likely in these conditions the B cells do not need to acquire the antigens as each BCR on B cell surface has the equal chance or is probably quarantined to engage the antigens without the needs for B cells to actively acquire the antigens (Reth and Wienands, 1997; Kurosaki et al., 2010). However, in these early studies, it is still widely observed that the $B$ cells showed dramatic and ordered morphological changes over time after encountering the free mobile soluble antigens. In resting B cells, the BCRs evenly distribute on cell surface, however when activated by free mobile soluble antigens, BCRs will initially go patching and ultimately go capping, suggesting that BCRs are polarized (Puffer et al., 2007). Early studies also have demonstrated that these morphological changes are required to trigger $B$ cell activation (Phee 
A
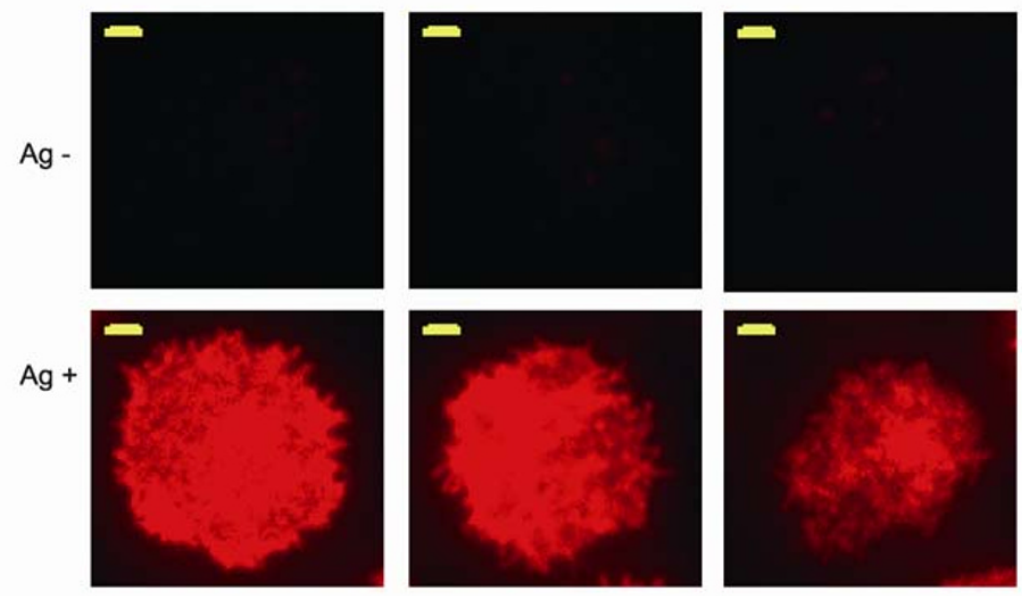

B

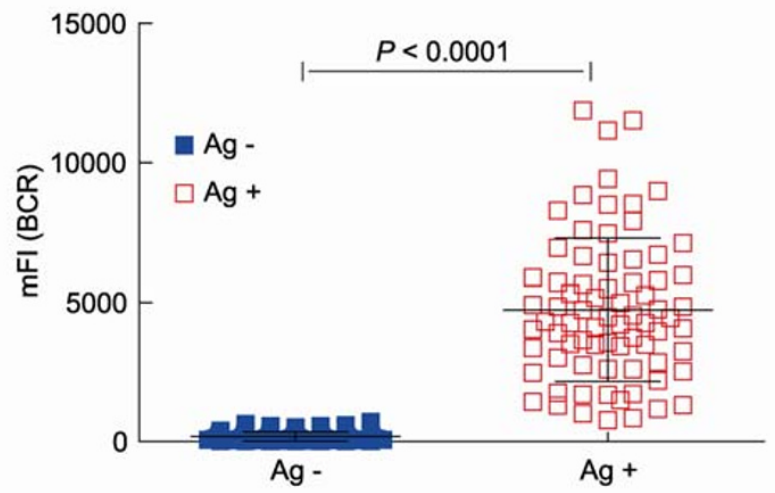

C

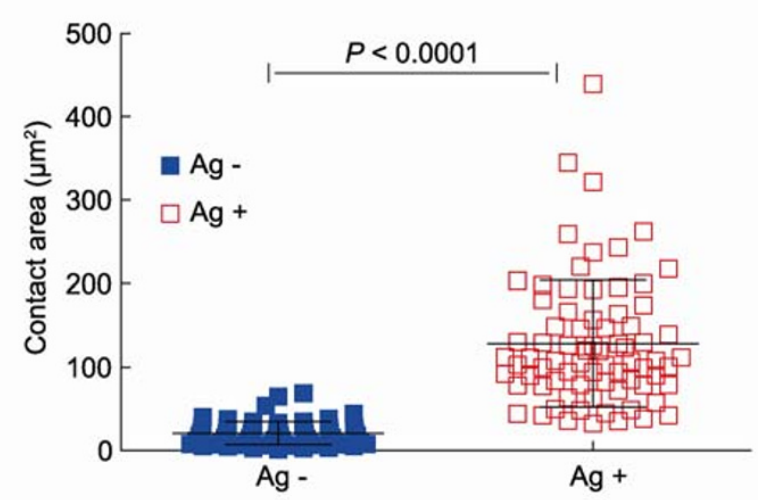

D
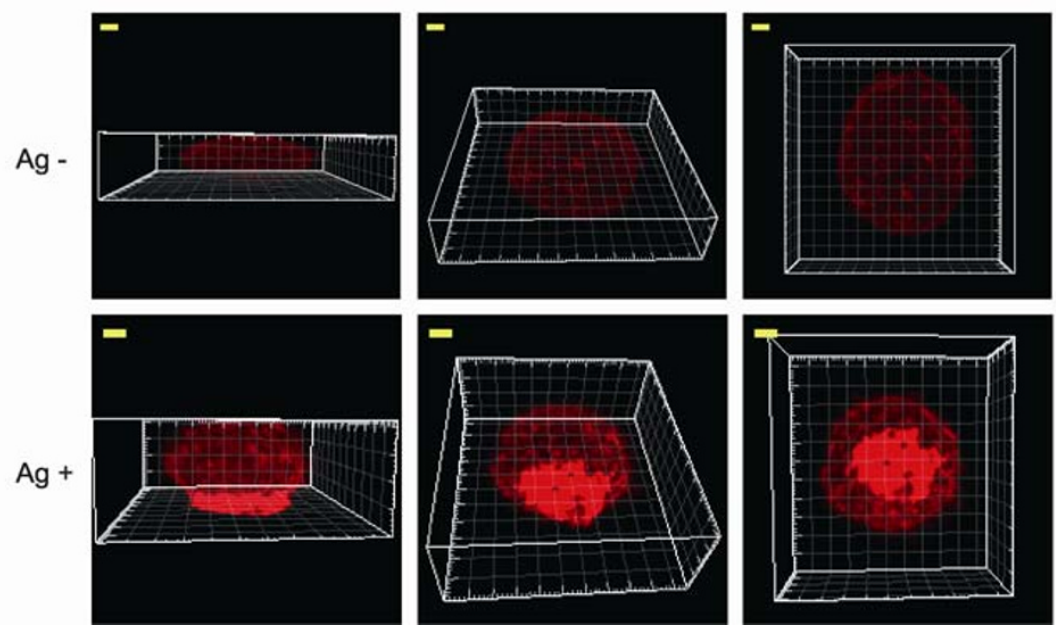

Figure 3. The state of $B$ cells 10 min after encountering fixed immobile antigens. (A) Shown are representative TIRF images of Ramos cells that were pre-stained with Alexa568-Fab anti-lgM and were placed on the glass coated with or without antigen. The scale bar is $1.6 \mu \mathrm{m}$. (B) The mean $\mathrm{FI}(\mathrm{mFI})$ of BCR microclusters within the B cell's contact area with the cover slides. (C) The size of the contact area. In $\mathrm{B}$ and $\mathrm{C}$, each dot represents one cell analyzed in three independent experiments, and bars represent means \pm SD. Two-tailed $t$ tests were performed for the statistical comparisons. (D) Reconstructed 3D confocal images of Ramos cells placed on the cover slide coated with or without antigen. The scale bar is $1.6 \mu \mathrm{m}$. The 3D images are shown from three different views: side (left panel), $45^{\circ}$ angle (middle panel) and top (right panel) (see more detail in Supplemental video 2). 
A
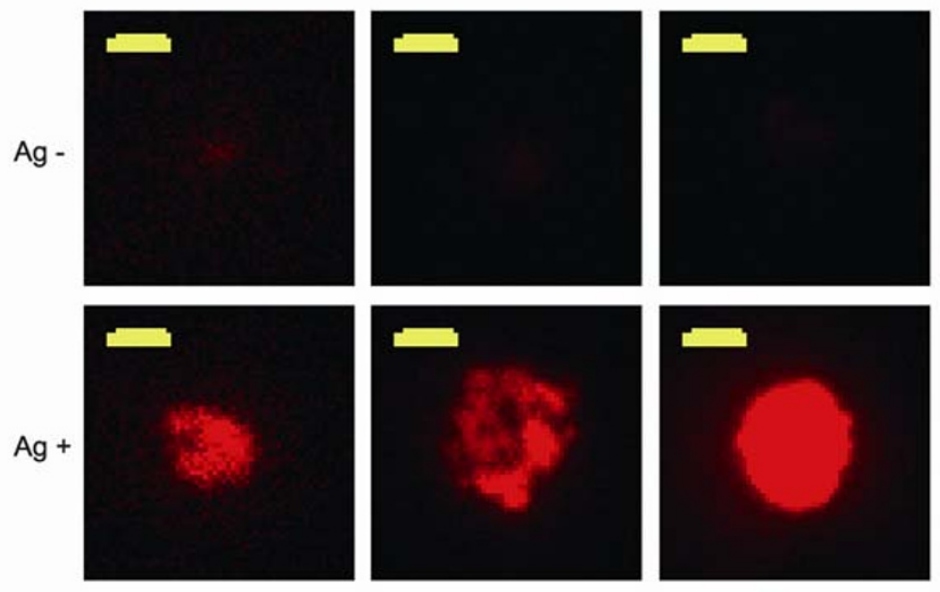

B

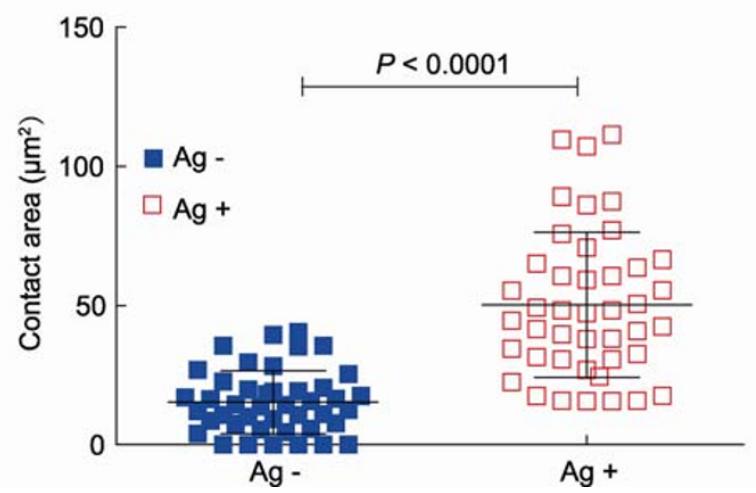

C

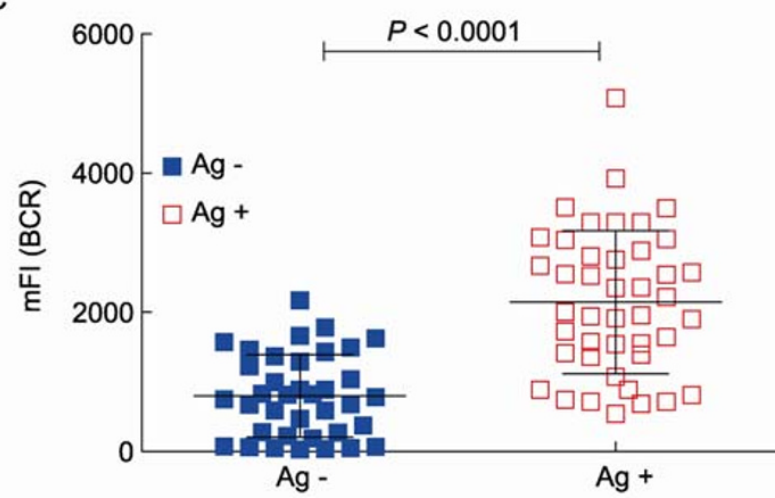

Figure 4. The state of B1-8 primary B cells encountering fixed immobile antigens. (A) Shown are TIRF images of B1-8 cells that were loaded on the glass coated with or without NIP25-BSA. The scale bar is $1.6 \mu \mathrm{m}$. (B and C) Given are the size of the contact area and the mean BCR FI of the contact area. Each dot represents one cell analyzed in three independent experiments, and bars represent means $\pm \mathrm{SD}$. Two-tailed $t$ tests were performed for the statistical comparisons.

et al., 2001; Gupta and DeFranco, 2003; Sohn et al., 2003; Blery et al., 2006; Puffer et al., 2007).

To our knowledge, it is not known whether or not the BCRs when encountering free mobile soluble antigen will undergo the dynamic microcluster growth feature as described in detail above. To address this question, we used spinning-disk confocal supported high resolution high speed live cell imaging experiments to visualize the morphological changes on the surface of Ramos and B1-8 B cells when encountering free mobile soluble antigens. As mentioned above, here we used goat anti-human IgM antibodies as surrogate antigen for Ramos B cells and NIP25-BSA for primary B1-8 B cells (Fig. $7 A$; Supplemental video 4). We quantified the mean fluorescence intensity $(\mathrm{mFI})$ of each BCR microcluster using the Imaris software. By comparing the $\mathrm{mFI}$ of $\mathrm{BCR}$ microclusters identified on the $B$ cells encountering the PBS control or free mobile soluble antigens in PBS buffer, we determined that free mobile soluble antigens as well can promote the BCR microclusters to get bigger and brighter (growth of BCR microclusters) compared to the PBS control group (Figs. 7B, 7C and 8; Supplemental video 5). Through these time lapse imaging studies, we determined that indeed, the growth of BCR microclusters in FI and size is substantial (Supplemental video 4). We observed the $\mathrm{mFI}$ of $\mathrm{BCR}$ microclusters is obviously bigger in the middle and late time points (for example, 7-9 and 16-18 $\mathrm{min}$ ) than the value in the earl time points (for example, 1-3 and 4-6 min) (Fig. 7B; Supplemental video 4). We got some similar observations when imaging the response of primary B1-8 B cells encountering free mobile soluble NIP25-BSA antigens (Fig. 7D).

These experiments suggest that the dynamic nature of BCR microclusters is a universal mechanism for $B$ cells to response to antigens with three different motion features: free mobile soluble antigens, lateral mobile membrane bound antigens, and fixed immobile antigens.

\section{DISCUSSION}

B cell plays central role in antibody response. On sensing cognate antigens, B cells get activated, proliferate and dif- 
A

$\mathrm{Ag}-$

$\mathrm{Ag}+$
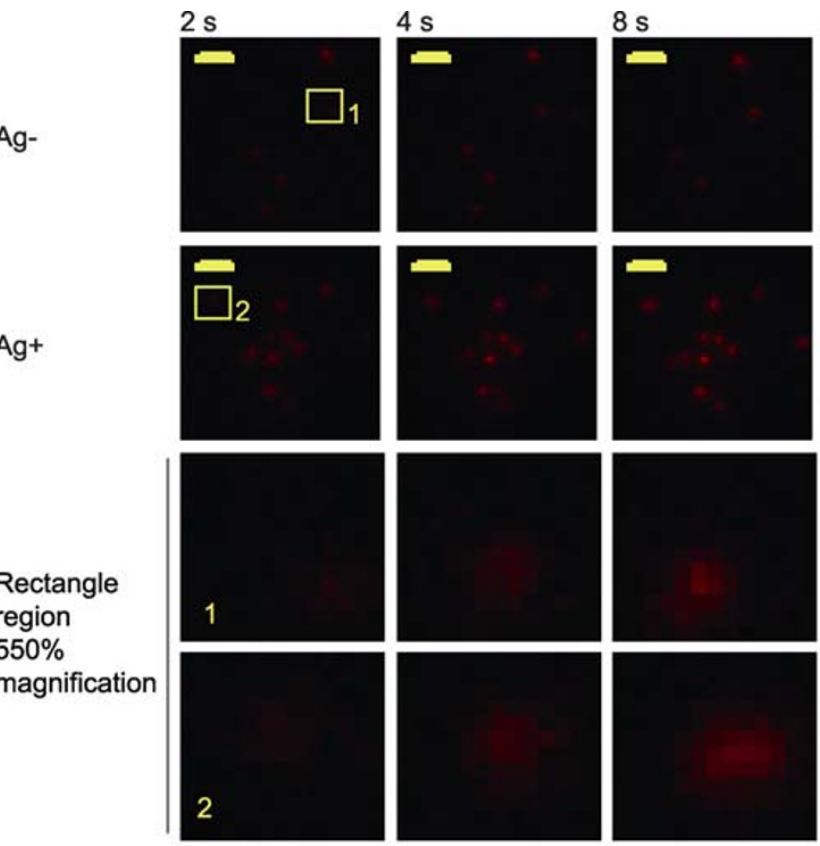

$16 s$
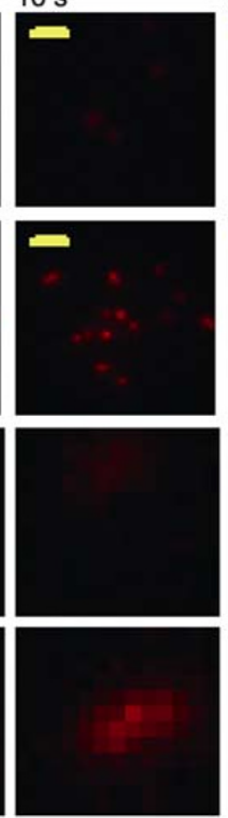

$32 \mathrm{~s}$
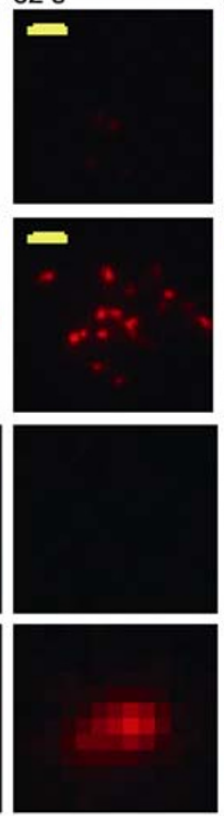

$60 \mathrm{~s}$
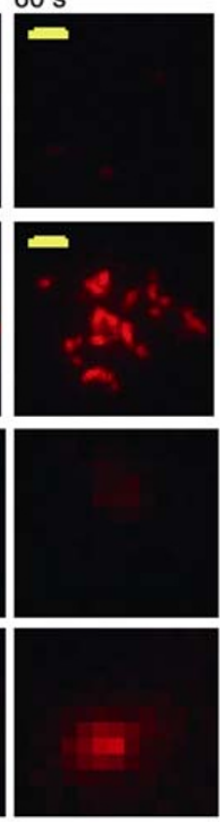

B

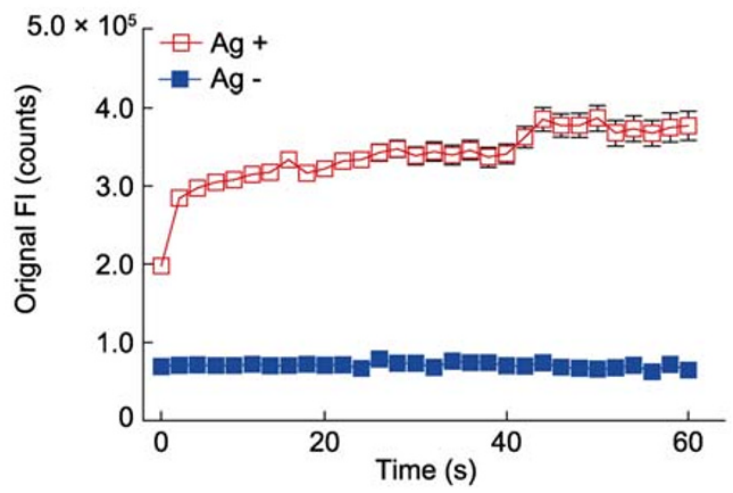

D

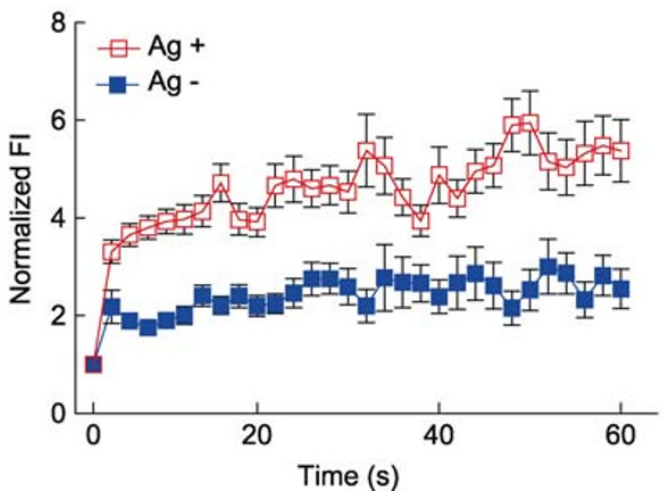

C

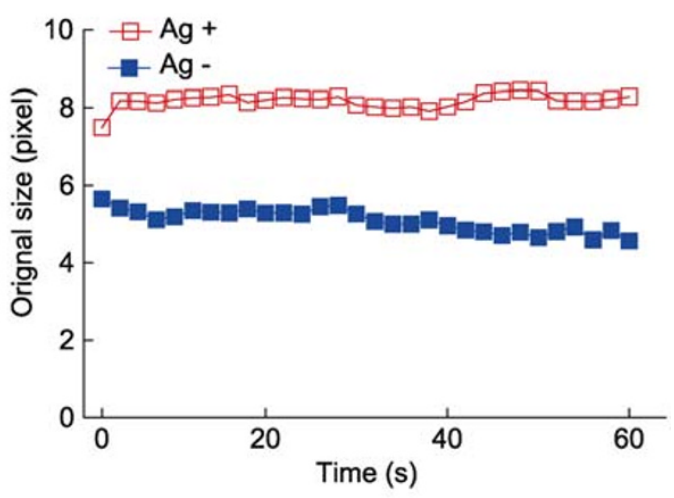

E

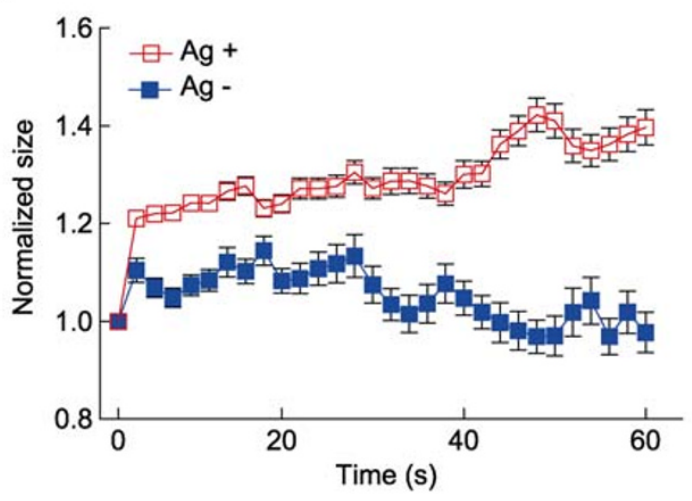

Figure 5. The response of Ramos cells encountering lateral mobile antigens. (A) Given are TIRF images of Ramos cells that were placed on fluid planar lipid bilayer with or without antigens at the indicated time points (see detail in Supplemental Video 3). Scale bar is $1.6 \mu \mathrm{m}$. Typical microclusters in the images indicated by the yellow box 1 and box 2 are shown at $550 \%$ magnification for better resolution. (B-E) The original FI (B), original size (C), normalized FI (D) and normalized size (E) of BCR microclusters on Ramos cells placed on fluid planar lipid bilayers lacking antigen or containing antigen at a concentration of $50 \mu \mathrm{g} / \mathrm{mL}$. The data represent means \pm SEM of at least 22 thousands BCR microclusters acquired from three independent experiments. 
A
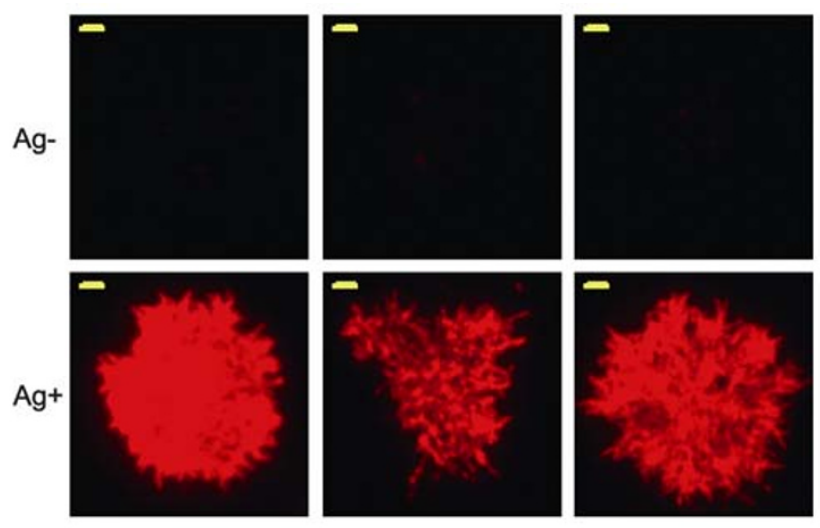

B

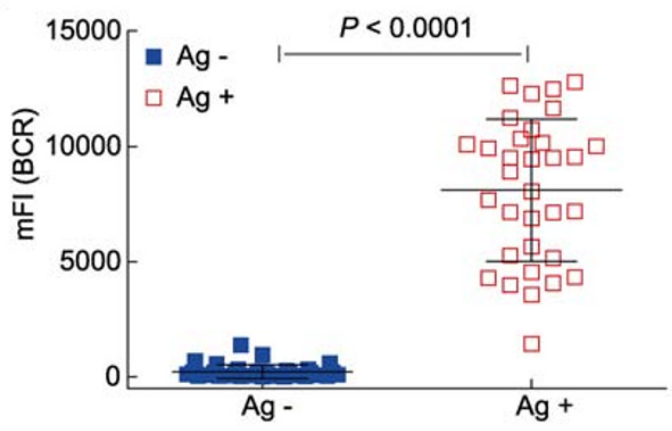

C

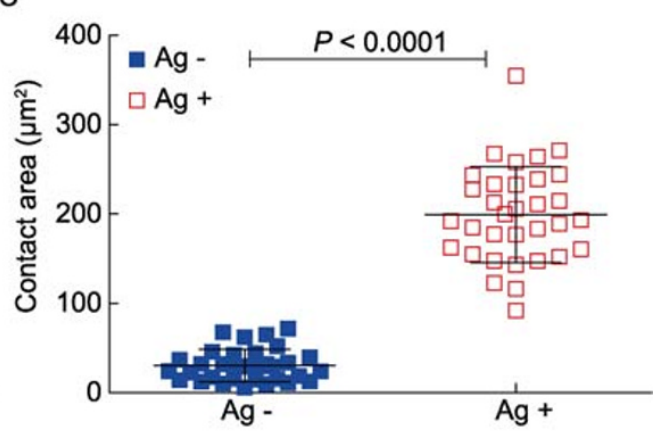

D
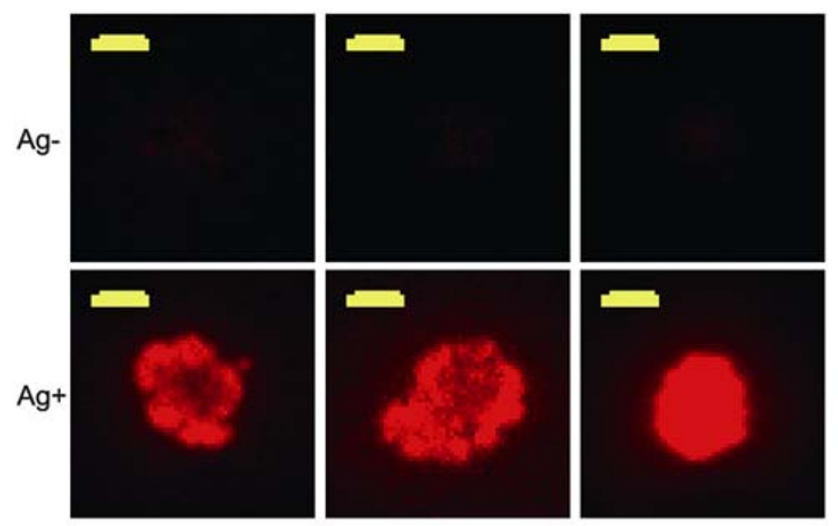

E
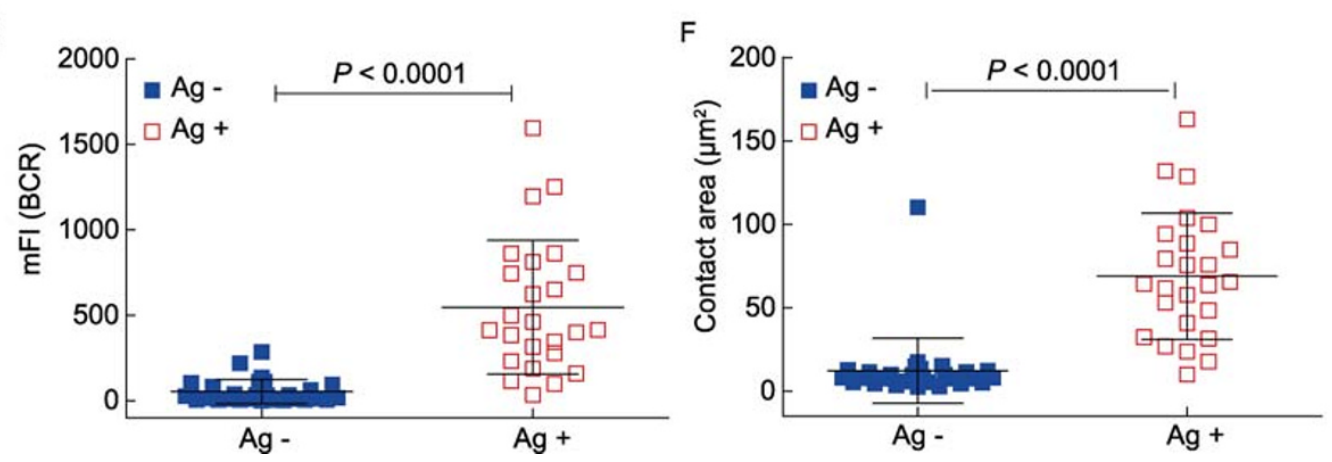

Figure 6. The state of Ramos or B1-8 B cells 10 min after encountering lateral mobile antigens. (A and D) Given are TIRF images of Ramos cells $(A)$ or $B 1-8$ cells $(D)$ placed on lipid bilayer with or without antigen. The scale bar is 1.6 $\mu \mathrm{m}$. (B, C, E and F) Given are the mean BCR FI (B and $E$ ) and size $(C$ and $F)$ of either Ramos ( $B$ and $C$ ) or B1-8 (E and F) B cells within the contact area. Each dot represents one cell analyzed in three independent experiments, and bars represent means \pm SD. Two-tailed $t$ tests were performed for the statistical comparisons. 
A
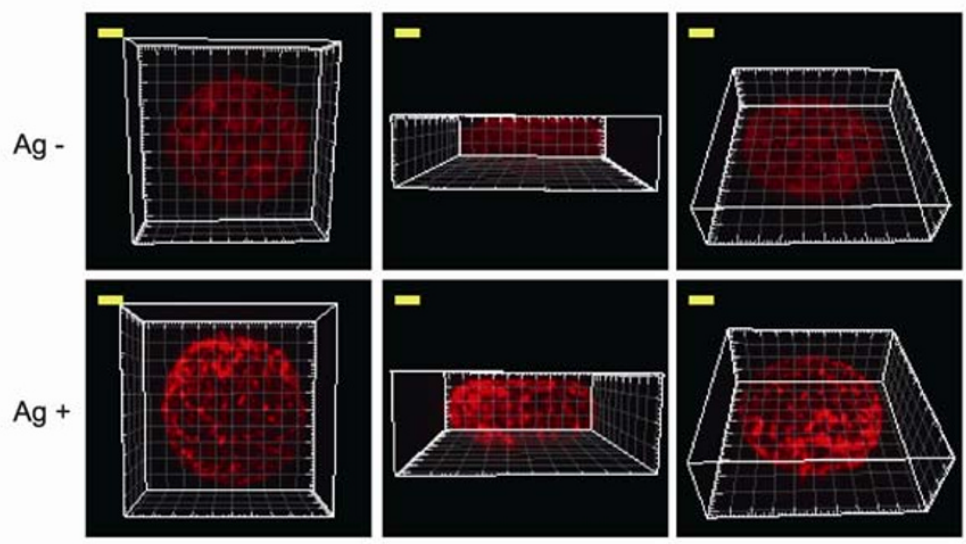

B

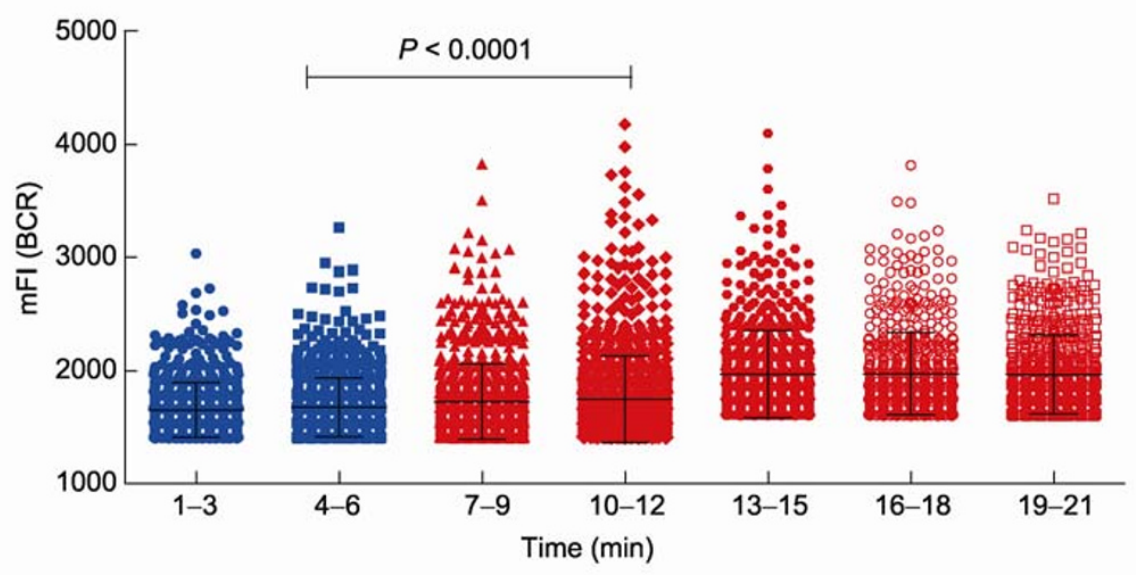

C

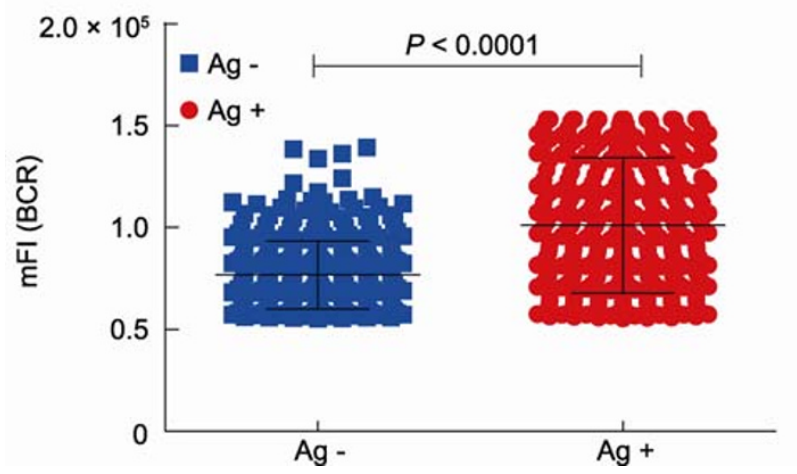

D

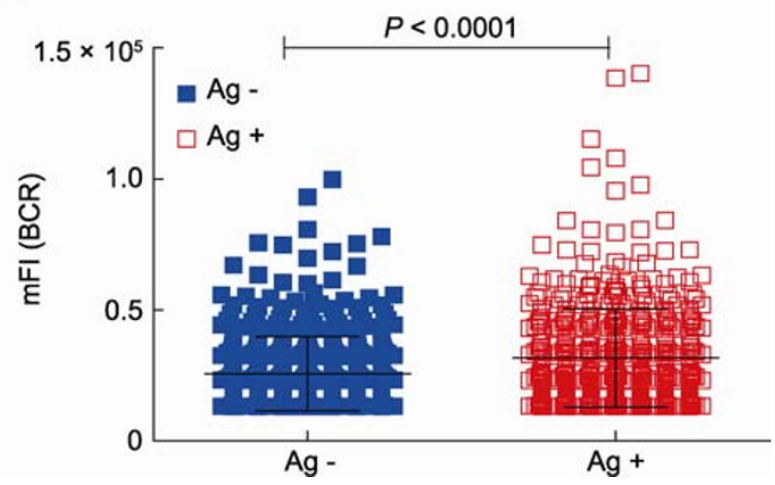

Figure 7. The response of $B$ cells encountering free mobile soluble antigens. (A) Reconstructed $3 D$ confocal images of Ramos cells encountering with mobile soluble antigens, goat anti-Human lgM, in PBS or PBS control only. The scale bar is $1.6 \mu \mathrm{m}$. The 3D images are shown from three different views: top (left panel), side (middle panel) and $45^{\circ}$ angle (right panel). Also see Supplemental video 5. (B) The mean FI of BCR microclusters on the surface of Ramos cells at indicate time periods after encountering mobile soluble antigens. Also see supplemental video 4. ( $C$ and $D)$ The mean FI of BCR microclusters on Ramos cells (C) or B1-8 cells (D) 10 min after encountering soluble antigen or PBS control. Each dot represents one BCR microcluster and bars represent means $\pm \mathrm{SD}$. Two-tailed $t$ tests were performed for the statistical comparisons.

ferentiate to plasma cells and memory B cells. All these complicated and ordered $B$ cell responses get started with the $B$ cell's sense and subsequent acquisition of foreign an- tigens. Thus it is of vital importance to investigate the mechanisms of how $B$ cells acquire antigens. The antigens can show great diversity by their presentation formats. Al- 


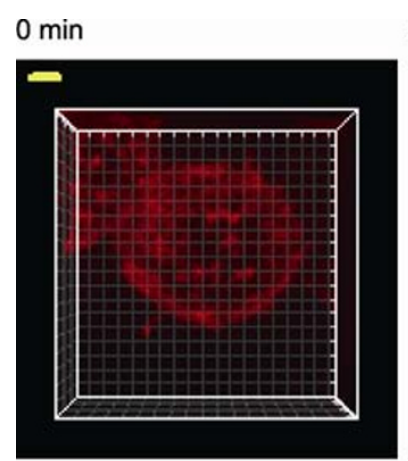

3 min

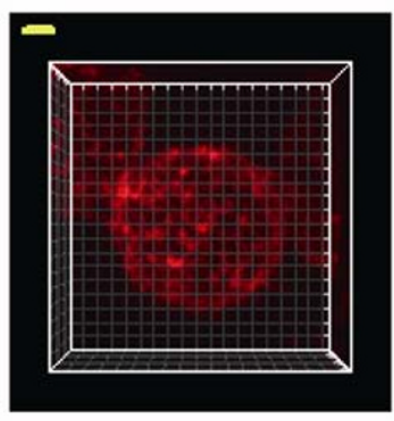

$12 \mathrm{~min}$

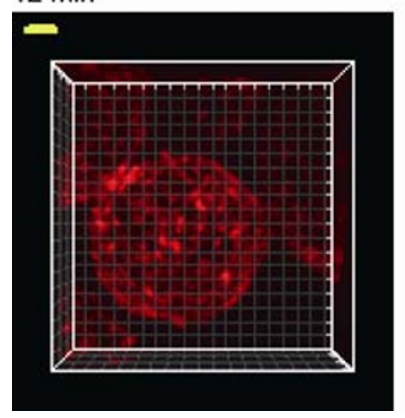

\begin{abstract}
$15 \mathrm{~min}$
\end{abstract}

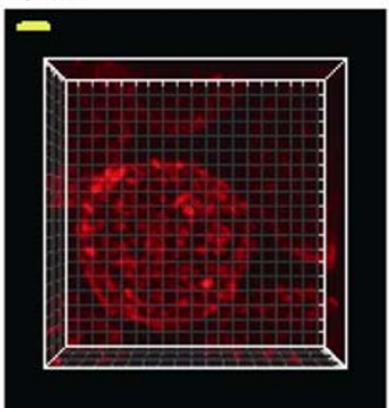

$6 \mathrm{~min}$

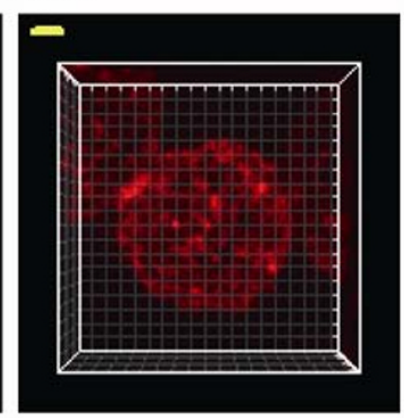

$18 \mathrm{~min}$

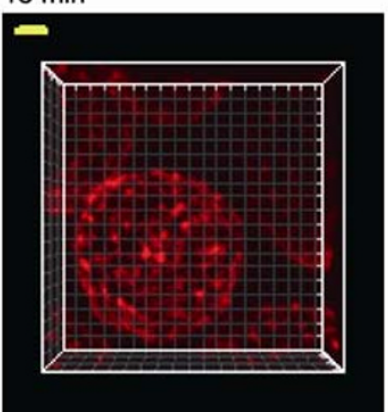

$9 \min$

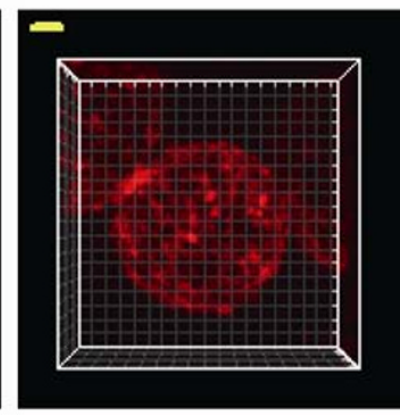

$21 \mathrm{~min}$

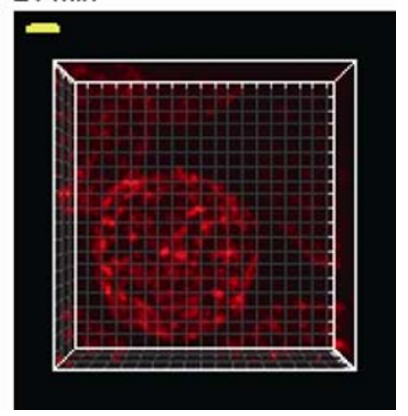

Figure 8. The real time response in $\mathbf{2 1} \mathrm{min}$ of one representative $B$ cell encountering free mobile soluble antigens as recorded in 3D time lapse images. Demonstrated is the obvious formation and dynamic growth of BCR microclusters over time on the surface of Ramos B cells. The scale bar is $1.6 \mu \mathrm{m}$. Also see Supplemental video 5 .

though it has been widely observed that the presentation format of antigens can dramatically influence antibody responses, it is until very recent years, with the help of the advanced high resolution high speed live cell imaging techniques, B cell immunologists begin to appreciate that there are dynamic and ordered molecule events to grant B cells the ability to acquire the antigens. Interestingly, the early studies of others and us demonstrated that the ability of B cells to acquire foreign antigens is proportionally translated into the downstream strength of B cell activation effects, suggesting the importance of developing new vaccine antigen formats that can induce the best of B cell's response to acquire vaccine antigen.

In this study, we focused on the state of the Brownian diffusion mobility of antigens. Using this criteria, there are three presentation formats of antigens: free mobile soluble antigens, lateral mobile membrane bound antigens, and fixed immobile antigens. Free soluble antigens can do three dimensional $(X$, $Y$ and $Z$ ) free Brownian motion; lateral mobile membrane bound antigens can do two dimensional ( $X$ and $Y$, but not $Z$ ) free Brownian motion; while fixed immobile antigens cannot do Brownian motion in each dimension. We asked how the antigens presented with very different mobility format can affect the B cell's antigen acquisition abilities, and how the BCR microclusters formation and dynamic growth feature can be affected.

We addressed these questions using a variety of cut- ting-edge high resolution high speed live cell imaging techniques supported by TIRFM and spinning-disk confocal microscope. We found that fixed immobile antigens, lateral mobile membrane bound antigens and free mobile soluble antigen seem to behavior similarly in terms of inducing the formation of BCR microclusters and the subsequent driving of BCR microcluster growth feature. Specifically, we found that both fixed immobile antigens coated on the surface of cover-slides and lateral mobile membrane bound antigens tethered on the surface of planar lipid bilayers can strongly stimulate the B cells to show the early membrane molecule events to acquire the antigens. For each type of antigen presentation formats, B cells spread much broader to search for more antigens, and to accumulate BCRs into the cell's contact interface compared to the antigen free control, saying the presentation format of both fixed immobile antigens and lateral mobile membrane bound antigens can serve as good BCR stimulus.

Our experiment imaging B cell's responses placed on cover-slides coated with antigen suggest that the B cell did spread over the antigen containing surface. However we observed very subtle contraction responses following the spreading behavior of the $B$ cells. These observations are different from the obvious spreading and contraction responses of B cells placed on planar lipid bilayers containing antigens as reported in early studies (Fleire et al., 2006; Tolar et al., 2009a). We speculate that the two-phase spreading 
and contraction responses probably are not the only behavior for $B$ cells to acquire antigens, although indeed it is a very efficient manner for $B$ cells to acquire sufficient amount of antigens in short amount of time. We guess that an efficient and obvious two-phase spreading and contraction response of $B$ cells can only show up at the optimal stimulating conditions. It will be very interesting to investigate how to achieve such condition. These pieces of information will be informative for potent and effective vaccine constructs.

How is the case in non-optimal stimulating conditions? If the $B$ cells do not show the two-phase spreading and contraction, how $B$ cells can acquire the BCR-antigens? Here, we propose that $B$ cells can acquire antigens through the manner of the dynamic growth behavior of BCR microclusters. Not surprisingly, we provide evidence that both fixed immobile antigens and lateral mobile membrane bound antigens can induce the formation and subsequent growth feature of BCR microclusters. How is the case of the free mobile soluble antigen, which is probably the most commonly used format of antigen in B cell biology studies? Early biochemical studies use this type of antigens in sufficient concentration to address the BCR signaling pathway molecules. By nature, the free mobile soluble antigen shows some very unique feature that does not possess by any other formats of antigens. Each antigen molecule of free mobile soluble antigen can do 3-dimensional free Brownian motion in solution. Most likely in this case the $B$ cells do no need to acquire the antigens as each BCR on B cell surface has the equal chance or is probably quarantined to engage the antigens without the needs for $B$ cells to actively acquire the antigens. Very unexpectedly, when imaging the behavior of $B$ cells when encountering the most commonly used free mobile soluble antigens, we could demonstrate the growth of BCR microclusters as well with the help of the high resolution spinning disk confocal microscope. Although, it is quite clear that the growth of BCR microclusters on B cells when encountering free mobile antigens is much milder compared to the case of fixed immobile antigens or lateral mobile antigens. These observations suggest that soluble antigen might be a weaker stimulator to cluster BCRs, consistent with the reports that antigens presented in lateral mobile membrane bound formats are more potent than soluble antigens as an immunogenic construct.

In summary, all these experiments suggest that the dynamic nature of BCR microclusters is a universal mechanism for $B$ cells to response to antigens in each presentation format: free mobile soluble antigens, lateral mobile membrane bound antigens, and fixed immobile antigens.

\section{MATERIALS AND METHODS}

Mice, cells, antibodies and antigens

B1-8 primary $B$ cells were isolated from spleens of $\operatorname{lgH}^{\text {B1-8/B1-8 }} \operatorname{lgk}^{-1-}$ transgenic mice as reported in our previous studies (Liu et al., 2010a). Purified unconjugated Fab goat anti-human $\operatorname{lgM}$, and biotin-conjugated goat anti-human IgM were purchased from Jackson ImmunoResearch. Fluorophore-conjugated anti-human IgM constant regions or anti-mouse IgM were purchased from Jackson ImmunoResearch. BSA conjugated 1:25 with 4-hydroxy-5-iodo-3-nitrophenyl acetyl (NIP) (NIP25-BSA) and NIP1-His were purchased from Biosearch Technologies.

\section{Preparation of fixed immobile antigen on glass cover slides}

Glass coverslips (Thermo Fisher Scientific) were cleaned by strip buffer $\left(\mathrm{H}_{2} \mathrm{SO}_{4}: \mathrm{H}_{2} \mathrm{O}_{2}=7: 3\right)$, washed, and dried. Anti-human IgM antibody or NIP25-BSA were added at $50 \mathrm{ug} / \mathrm{ml}$ to the slides. Incubated at $37^{\circ} \mathrm{C}$ for $30 \mathrm{~min}$. After washing, cover slides were blocked with $5 \%$ $\mathrm{BSA}$ at $37^{\circ} \mathrm{C}$ for $2 \mathrm{~h}$. After washing, the slices were ready to be used for TIRFM imaging.

\section{Preparation of fluid planar lipid bilayers and lateral mobile antigens}

Planar fluid lipid bilayers were prepared as previously described (Liu et al., 2010a). In brief, Biotin-containing planar lipid bilayers were prepared with 99\% 1,2-dioleoyl-sn-glycero-3-phosphocholine (DOPC) and 1\% 1,2-Dioleoyl-sn-Glycero-3-phosphoethanolamine-cap-biotin (DOPE-capbiotin) (Avanti Polar Lipids). Unilamellar vesicles were formed by sonication of the mixed lipids and clarified by ultracentrifugation and filtering. Glass coverslips were cleaned by strip buffer $\left(\mathrm{H}_{2} \mathrm{SO}_{4}: \mathrm{H}_{2} \mathrm{O}_{2}=7: 3\right)$, washed, and dried. Lipid bilayers were prepared from $0.2 \mathrm{mmol} / \mathrm{L}$ lipid unilamellar vesicle solutions on the coverslip attached to the bottom of Labtek chambers. $50 \mathrm{nmol} / \mathrm{L}$ streptavidin was incubated with biotin-containing lipid bilayer at room temperature for $10 \mathrm{~min}$. After washing, $50 \mu \mathrm{g} / \mathrm{mL}$ biotinylated Abs, biotin-antihuman $\lg G+\lg M$ specific for human $\lg M$ were bound to the planar lipid bilayer. After washing, lipid bilayer was blocked with $5 \%$ BSA in $37^{\circ} \mathrm{C}, 5 \% \mathrm{CO}_{2}$, for $30 \mathrm{~min}$. Ni-NTA-containing lipid bilayers were prepared using the DOPC lipid and 1,2-dioleoylsn-glycero-3-[N(5-amino-1- carboxypentyl) imino-diacetic acid]-succinyl (nickel salt) (DOGS-Ni- NTA; Avanti Polar Lipids, Alabaster, AL) with 90\% DOPC and 10\% DOGS-Ni-NTA. $10 \mathrm{nmol} / \mathrm{L}$ NIP1-His was incubated with Ni-NTA-containing lipid bilayers at room temperature for $10 \mathrm{~min}$. After washing, the lipid bilayers were ready for TIRFM.

\section{Time-lapse live-cell imaging by TIRFM and spinning disk confocal microscope}

Immediately after the encountering of antigens with different motion feature, the response of $B$ cells and behavior of BCR microclusters were imaged by TIRFM or Spinning disk confocal fluorescence microscope. Time lapse images were acquired at $37^{\circ} \mathrm{C}$ on a heated stage by an Olympus IX-81 microscope supported by a TIRF port, ANDOR iXon+ DU-897D electron-multiplying EMCCD camera, Olympus 100X objective lens. The acquisition was controlled by ANDOR IQ2 software. The exposure time was $100 \mathrm{~ms}$ unless spe- 
cially indicated. $561 \mathrm{~nm}$ laser was used. Spinning disk confocal images were acquired by the same Olympus IX-81 microscope supported by the yokogawa spinning disk sets. Images were analyzed by Image Pro Plus (Media Cybernetics), Image J (NIH, U.S.), or Matlab (Mathworks) software as indicated.

\section{Single BCR microcluster tracking and 2D-Gaussian analyses}

Single BCR microcluster tracking and 2D-Gaussian analyses were performed as we have reported previously (Tolar et al., 2009a; Liu et al., 2010a). Briefly, each BCR microcluster in time-lapse TIRF images were processed by means of least square fitting of a 2D Gaussian function (Holtzer et al., 2007). For each microcluster, the fit yields the integrated $\mathrm{FI}$ and generalized FWHM of the intensity distribution in the $x$ and $y$ direction.

\section{ACKNOWLEDGEMENTS}

We thank Rui Shi and Lei Huang at the Center of Biomedical Analysis, Tsinghua University, for their technique support on spinning disk confocal, and TIRFM imaging experiments. We thank Dr. Susan K. Pierce at NIAID-NIH for providing experimental materials. This work is supported by funds from Tsinghua University and Center for Life Sciences.

\section{ABBREVIATIONS}

APC, antigen presenting cell; BCR, B cell receptor; FWHM, full width at half-maximum peak height; IS, immunological synapse; $\mathrm{mFI}$, mean fluorescence intensity; TIRFM, total internal reflection fluorescence microscopy

\section{REFERENCES}

Bachmann, M. F., Rohrer, U. H., Kundig, T. M., Burki, K., Hengartner, H., and Zinkernagel, R. M. (1993). The influence of antigen organization on B cell responsiveness. Science 262, 1448-1451.

Batista, F. D., and Harwood, N. E. (2009). The who, how and where of antigen presentation to B cells. Nat Rev Immunol 9, 15-27.

Batista, F.D., Iber, D., and Neuberger, M.S. (2001). B cells acquire antigen from target cells after synapse formation. Nature 411 , 489-494.

Blery, M., Tze, L., Miosge, L. A., Jun, J. E., and Goodnow, C. C. (2006). Essential role of membrane cholesterol in accelerated BCR internalization and uncoupling from NF-kappa B in B cell clonal anergy. J Exp Med 203, 1773-1783.

Carrasco, Y. R., and Batista, F. D. (2007). B cells acquire particulate antigen in a macrophage-rich area at the boundary between the follicle and the subcapsular sinus of the lymph node. Immunity 27 , 160-171.

Depoil, D., Fleire, S., Treanor, B. L., Weber, M., Harwood, N. E., Marchbank, K. L., Tybulewicz, V. L., and Batista, F. D. (2008). CD19 is essential for B cell activation by promoting B cell receptor-antigen microcluster formation in response to membrane-bound ligand. Nat immunol 9, 63-72.

Fleire, S. J., Goldman, J. P., Carrasco, Y. R., Weber, M., Bray, D., and Batista, F. D. (2006). B cell ligand discrimination through a spreading and contraction response. Science 312, 738-741.

Gupta, N., and DeFranco, A.L. (2003). Visualizing lipid raft dynamics and early signaling events during antigen receptor-mediated B-lymphocyte activation. Mol Biol Cell 14, 432-444.

Holtzer, L., Meckel, T., and Schmidt, T. (2007). Nanometric three-dimensional tracking of individual quantum dots in cells. Appl Phys Lett 90, 053902-053904.

Junt, T., Moseman, E. A., lannacone, M., Massberg, S., Lang, P. A., Boes, M., Fink, K., Henrickson, S. E., Shayakhmetov, D. M., Di Paolo, N. C., et al. (2007). Subcapsular sinus macrophages in lymph nodes clear lymph-borne viruses and present them to antiviral B cells. Nature 450, 110-114.

Kurosaki, T., Shinohara, H., and Baba, Y. (2010). B cell signaling and fate decision. Annu Rev Immunol 28, 21-55.

Kusumi, A., Nakada, C., Ritchie, K., Murase, K., Suzuki, K., Murakoshi, H., Kasai, R. S., Kondo, J., and Fujiwara, T. (2005). Paradigm shift of the plasma membrane concept from the two-dimensional continuum fluid to the partitioned fluid: high-speed single-molecule tracking of membrane molecules. Annu Rev Biophys Biomol Struct 34, 351-378.

Kusumi, A., Shirai, Y. M., Koyama-Honda, I., Suzuki, K. G., and Fujiwara, T. K. (2010). Hierarchical organization of the plasma membrane: investigations by single-molecule tracking vs. fluorescence correlation spectroscopy. FEBS Lett 584, 1814-1823.

Lin, K.B.L., Freeman, S.A., Zabetian, S., Brugger, H., Weber, M., Lei, V., Dang-Lawson, M., Tse, K. W. K., Santamaria, R., Batista, F. D., et al. (2008). The Rap GTPases regulate B cell morphology, immune-synapse formation, and signaling by particulate $B$ cell receptor ligands. Immunity 28, 75-87.

Liu, W., Meckel, T., Tolar, P., Sohn, H. W., and Pierce, S. K. (2010a). Antigen affinity discrimination is an intrinsic function of the $B$ cell receptor. J Exp Med 207, 1095-1111.

Liu, W., Meckel, T., Tolar, P., Sohn, H. W., and Pierce, S. K. (2010b). Intrinsic properties of immunoglobulin IgG1 isotype-switched B cell receptors promote microclustering and the initiation of signaling. Immunity 32, 778-789.

Liu, W., Sohn, H. W., Tolar, P., and Pierce, S. K. (2010c). It's all about change: the antigen-driven initiation of B-cell receptor signaling. Cold Spring Harb Perspect Biol 2, a002295.

Liu, W., Won Sohn, H., Tolar, P., Meckel, T., and Pierce, S. K. (2010d). Antigen-induced oligomerization of the B cell receptor is an early target of FcgRIIB inhibition. J Immunol 184, 1977-1989.

Phan, T. G., Grigorova, I., Okada, T., and Cyster, J. G. (2007). Subcapsular encounter and complement-dependent transport of immune complexes by lymph node B cells. Nat Immunol 8, 992-1000.

Phee, H., Rodgers, W., and Coggeshall, K. M. (2001). Visualization of negative signaling in $\mathrm{B}$ cells by quantitative confocal microscopy. Mol Cell Biol 21, 8615-8625.

Pierce, S. K., and Liu, W. (2010). The tipping points in the initiation of B cell signalling: how small changes make big differences. Nat Rev Immunol 10, 767-777.

Puffer, E.B., Pontrello, J. K., Hollenbeck, J. J., Kink, J. A., and Kiessling, L. L. (2007). Activating B cell signaling with defined multivalent ligands. ACS Chem Biol 2, 252-262.

Qi, H., Egen, J. G., Huang, A. Y., and Germain, R. N. (2006). Extrafollicular activation of lymph node $B$ cells by antigen-bearing dendritic cells. Science 312, 1672-1676. 
Reth, M., and Wienands, J. (1997). Initiation and processing of signals from the B cell antigen receptor B. Annu Rev Immunol 15, 453-479.

Schamel, W. W., and Reth, M. (2000). Monomeric and oligomeric complexes of the B cell antigen receptor. Immunity 13, 5-14.

Sohn, H. W., Gu, H., and Pierce, S. K. (2003). Cbl-b negatively regulates $B$ cell antigen receptor signaling in mature $B$ cells through ubiquitination of the tyrosine kinase Syk. J Exp Med 197, 1511-1524.

Tolar, P., Hanna, J., Krueger, P. D., and Pierce, S. K. (2009a). The constant region of the membrane immunoglobulin mediates $B$ cell-receptor clustering and signaling in response to membrane antigens. Immunity 30, 44-55.

Tolar, P., Sohn, H. W., Liu, W., and Pierce, S. K. (2009b). The molecular assembly and organization of signaling active B cell receptor oligomers. Immunol Rev 232, 34-41.
Tolar, P., Sohn, H. W., and Pierce, S. K. (2005). The initiation of antigen-induced $B$ cell antigen receptor signaling viewed in living cells by fluorescence resonance energy transfer. Nat immunol 6 , 1168-1176.

Treanor, B., and Batista, F. D. (2010). Organisation and dynamics of antigen receptors: implications for lymphocyte signalling. Curr Opin Immunol 22, 299-307.

Treanor, B., Depoil, D., Gonzalez-Granja, A., Barral, P., Weber, M., Dushek, O., Bruckbauer, A., and Batista, F.D. (2010). The membrane skeleton controls diffusion dynamics and signaling through the B cell receptor. Immunity 32, 187-199.

Weber, M., Treanor, B., Depoil, D., Shinohara, H., Harwood, N. E., Hikida, M., Kurosaki, T., and Batista, F. D. (2008). Phospholipase $\mathrm{C}$-\{gamma\}2 and Vav cooperate within signaling microclusters to propagate $B$ cell spreading in response to membrane-bound antigen. J Exp Med 205, 853-868. 